\title{
Drug-Coated Balloons versus Everolimus-Eluting Stents in Patients with In-Stent Restenosis: A Pair-Wise Meta-Analysis of Randomized Trials
}

\author{
Nina Peng $\mathbb{D},{ }^{1}$ Wei Liu $\mathbb{D},{ }^{2}$ Zongzhuang Li $\mathbb{D}^{2},{ }^{2}$ Jun Wei $\mathbb{D},{ }^{1}$ Xuejun Chen $\mathbb{D}^{1},{ }^{1}$ \\ Wei Wang $\mathbb{D}^{1},{ }^{1}$ and Hao Lin $\mathbb{D}^{1}$ \\ ${ }^{1}$ Department of Internal Medicine, Guizhou Orthopedics Hospital, Guiyang, China \\ ${ }^{2}$ Department of Cardiology, Guizhou Provincial People's Hospital, Guiyang, China \\ Correspondence should be addressed to Wei Liu; liuw98@126.com
}

Received 21 July 2019; Revised 26 September 2019; Accepted 4 October 2019; Published 21 January 2020

Academic Editor: Ify R. Mordi

Copyright (c) 2020 Nina Peng et al. This is an open access article distributed under the Creative Commons Attribution License, which permits unrestricted use, distribution, and reproduction in any medium, provided the original work is properly cited.

Objective. This study aimed to compare the effectiveness of drug-coated balloons (DCB) with everolimus-eluting stents (EES) in the treatment of in-stent restenosis (ISR) and the differential relative effect of DCB in patients with drug-eluting stents (DES)-ISR and bare metal stents (BMS)-ISR. Background. The efficiency and safety of DCB and EES need to be assessed for the treatment of ISR. Methods. A systematic literature search was conducted using PubMed and EMBASE to identify all relevant studies. Angiographic results and clinical events were separately assessed. Subgroup meta-analyses were performed according to the type of restenosed stent. Results. Six randomized trials with 1134 patients were included. The overall pooled outcomes indicated that DCB was associated with lower minimum lumen diameter (mean difference $(M D)=-0.17,95 \% C I=-0.29$ to $-0.05, P=0.006$ ) and higher target lesion revascularization (risk ratio $(R R)=2.38,95 \% C I=1.36$ to $4.18, P=0.002$ ) than EES. However, the subgroup meta-analyses showed that DCB was inferior to EES only in DES-ISR patients, with lower minimum lumen diameter $(M D=-0.25,95 \% C I=-0.37$ to $-0.14, P<0.001)$, higher percent diameter stenosis $(M D=5.37,95 \% C I=1.33$ to $9.42, P=0.009)$, more binary restenosis $(R R=2.07,95 \% C I=1.20$ to $3.58, P=0.009)$, and higher incidence of target vessel revascularization $(R R=2.07,95 \% C I=1.22$ to $3.50, P=0.007)$ and target lesion revascularization $(R R=2.43,95 \% C I=1.28$ to $4.22, P=0.002)$. No differences in angiographic results and clinical events were found between DCB and EES in BMS-ISR patients. Conclusions. DCB was inferior to EES in DES-ISR and comparable in BMS-ISR in terms of angiographic results and clinical events.

\section{Introduction}

In-stent restenosis (ISR) is one of the main stumbling blocks for stent implantation in patients with coronary artery disease [1]. Although the medicines and stents had advanced prominently, ISR was still remarkable. Repeat revascularization for ISR accounts for $5-10 \%$ of patients undergoing percutaneous coronary intervention (PCI) with drug-eluting stents (DES) and for $20-30 \%$ after PCI with bare-metal stents (BMS) [1]. Many repeat revascularization strategies were performed in ISR patients, such as balloon angioplasty, BMS implantation, vascular brachytherapy, rotablation, DES implantation, or drug-coated balloon (DCB) angioplasty [2]. Among these strategies, DES implantation and DCB angioplasty are superior to other strategies [2]. The latest myocardial European revascularization guideline recommended DES implantation and DCB angioplasty for the treatment of ISR both of BMS or DES (class I, level A) [2].

Many trials had compared the efficacy and safety of DES and DCB for the treatment of ISR, but the results varied. A network meta-analysis suggests that PCI with everolimus-eluting stents (EES) and DCB angioplasty should be considered for the treatment of any type of coronary ISR [3]. In this network meta-analysis, EES was the most effective strategy for the treatment of ISR, with the lowest risks of restenosis and repeat revascularization compared with other treatments; DCB ranked second in terms of angiographic and clinical effectiveness [3]. However, this network meta-analysis only 
included two head-to-head comparative trials, RIBS IV and RIBS V, to synthesize the direct result of EES versus DCB. After this network meta-analysis, several trials compared head-tohead EES with DCB for the treatment of ISR with debated results [4-6]. The comparison of EES and DCB for the treatment of ISR remained controversial. Besides, previous studies demonstrated that DCB angioplasty was more effective in BMS-ISR than in DES-ISR. However, the differential relative efficacy between DCB and EES in patients with BMS-ISR and DES-ISR was still unknown.

Therefore, through a pair-wise meta-analysis of all relevant randomized evidence, this study aimed to directly compare DCB with EES for the treatment of ISR. Subgroup meta-analyses were performed to evaluate the differential relative effect of DCB in patients with DES-ISR and BMS-ISR.

\section{Materials and Methods}

The present systematic review and meta-analysis was performed in compliance with the recommendations of the PRISMA statement (Preferred Reporting Items for Systematic Reviews and Meta-Analyses) [7, 8].

2.1. Search Strategy. We searched PubMed and EMBASE (up to June 12,2019$)$ to identify all publications that compared DCB with DES for ISR therapy. The following terms were used by combining with proper logical connectors: "drugcoated balloon," "drug-eluting balloon," "drug-eluting stents," "everolimus-eluting stents," "randomized," "randomized," "randomly," "in-stent restenosis," and "coronary restenosis." Moreover, a manual search was performed by scanning the references of the identified articles to find potentially missing studies by the electronic searches.

2.2. Study Selection and Data Collection. The inclusion criteria of the present systematic review and meta-analysis were as follows: (1) a randomized controlled trial (RCT) was mandated, (2) patients were diagnosed with ISR, both for coronary arteries previously treated with BMS and DES, and (3) studies that compared DCB with EES.

The selection of study was strictly in compliance with the inclusion criteria. Two authors (NP and $W L$ ) independently assessed all potentially relevant studies. The selection process was carried out by crude screening to exclude irrelevant studies at the level of the title and abstract, and the remaining articles studies were double-checked by full text to achieve a final decision. A consensus was reached on all eligible studies between the two screening authors. Any discrepancies were resolved by discussion.

Two authors ( $Z L$ and $J W$ ) independently extracted all relevant information from eligible studies. A prespecified table that contained the relevant items was used to help with data collection.

2.3. Endpoints. In the present systematic review and metaanalysis, the different effects of DCB and EES for ISR therapy in BMS-ISR or DES-ISR patients were assessed. The angiographic results contained in-segment late lumen loss (LLL), in-segment minimal lumen diameter (MLD), percent diameter stenosis, and binary restenosis at follow-up angiography. The clinical events were target vessel revascularization (TVR), target lesion revascularization (TLR), myocardial infarction, and death. If two follow-up durations were reported, we chose the longer period.

2.4. Evaluation of Study Quality and Publication Bias. The Collaboration's "Risk of Bias" tool was also used to assess the risk of bias in the included studies.

2.5. Data Synthesis and Statistical Analysis. We conducted the present meta-analyses on angiographic results and clinical events of DCB and EES separately. The $I^{2}$ statistic was used to test statistical heterogeneity, with values of $>50 \%$ representing important heterogeneity, then a random-effects model was used to pool the effect sizes; While $I^{2} \leq 50 \%$ indicated insignificant heterogeneity, and a fixed-effects model was used to pool the effect sizes. Risk ratio (RR) was calculated as the effect size for endpoints with categorical data, and the mean difference (MD) with the $95 \%$ confidence interval (CI) was the effect size for endpoints with continuous data. Subgroup meta-analyses were performed according to the type of restenosed stent (BMS-ISR and DES-ISR). We performed sensitive analyses using leavingone-out approach. Trial sequential meta-analysis (TSA) was performed to assess the false positive errors (or type I errors) and false negative errors (or type II errors). Continuous data are presented as mean \pm standard deviation. Categorical data are presented as values and percentages.

All meta-analyses were pooled based on the Cochrane Handbook for Systematic Reviews of Interventions Version 5.1.0. Meta-analyses were conducted using the Review Manager software (version 5.3), and TSA were conducted using the TSA software (version 0.9.5.10 Beta).

\section{Results}

A total of 626 potential literature citations matched the systematic search strategy. Figure 1 presents in detail the study search and selection process. After strict selection, six trials with 1134 patients were included in the present pairwise meta-analysis [4-6, 9-11]. The TIS and SEDUCE trials were from a single center $[6,10]$, and the other four trials were performed at multiple centers. The follow-up durations of angiography ranged from 6 to 12 months. All the included trials reported 1-year clinical events, while RIBS IV, RIBS $\mathrm{V}$, and TIS trials further reported 3-year clinical events $[6,9,11-14]$. We used the 3-year follow-up clinical events to synthesize the final forest plots for the three trials [12-14].

The definition of restenosis was $>50 \%$ diameter stenosis on visual assessment in-stent and/or $<5 \mathrm{~mm}$ of the stent in all included trials, except for the SEDUCE trial, in which restenosis was defined as $>70-<100 \%$ for target lesion stenosis measured by quantitative coronary angiography. In the included six trials, the same DCB (paclitaxel-eluting balloon) was used. The clinical and lesions characteristics of included trials are summarized in Tables 1 and 2 . 


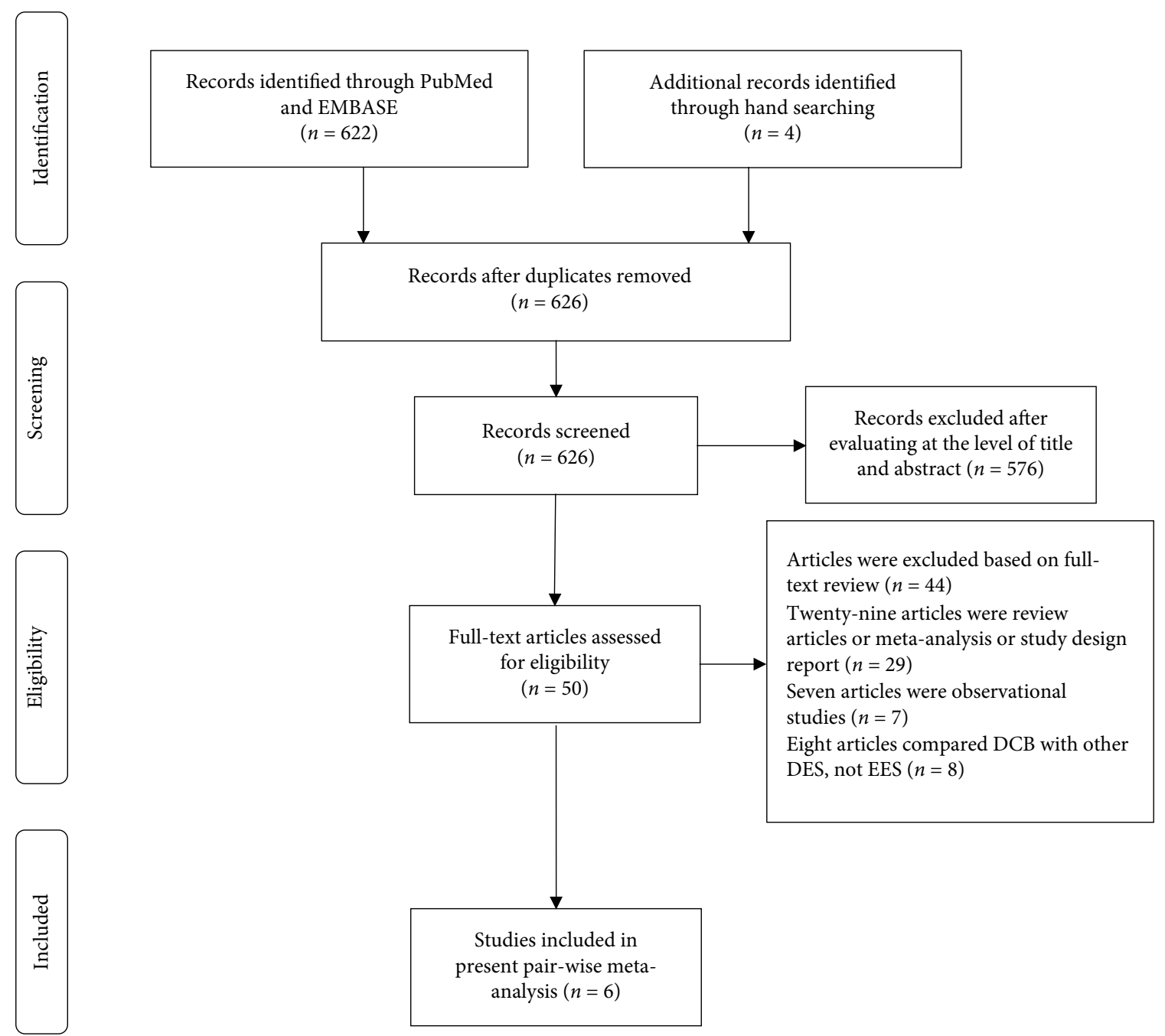

FIGURE 1: Flow chart of study selection.

3.1. Quality and Risk of Bias of the Included Studies. The summary assessment of risk of bias is shown in Figure 2. The quality was "high" because most information was obtained from included RCT studies with low risk of bias.

\subsection{Angiographic Results at Follow-Up}

3.2.1. MLD. All the six trials reported about the MLD. There were 569 patients in the DCB group and 565 patients in the EES group. The overall meta-analysis revealed that EES was superior to DCB in terms of MLD with MD of $-0.17 \mathrm{~mm}$ with important heterogeneity $(M D=-0.17$, $95 \% C I=-0.29$ to $-0.05, P=0.006, I^{2}=64 \%$, Table 3$)$. In the subgroup meta-analysis for DES-ISR, compared to EES, DCB was associated with smaller MLD $(M D=-0.25$, $95 \% C I=-0.37$ to $-0.14, P<0.001$, Table 3$)$. However, for BMS-ISR, MLD did not show significant difference between DCB and EES $(M D=-0.15,95 \% C I=-0.39$ to $0.09, P=0.22$, Table 3). The results remained stable using the leave-one-out approach after omitting any single trial from the analysis. The TSA showed before reaching the expected sample size, the result that EES was superior to DCB in terms of MLD was inconclusive (Data not shown).
3.2.2. LLL. LLL was evaluated in all six trials. The pooled result showed a similar LLL between the DCB group and EES group for ISR therapy, and significant heterogeneity was identified, i.e., between-trial heterogeneity, with $I^{2}=87 \%$ $\left(M D=-0.06,95 \% C I=-0.23\right.$ to $0.10, P=0.46, I^{2}=87 \%$, Table 3). For subgroup meta-analysis, DCB and EES had similar LLL for both DES-ISR and BMS-ISR $(M D=0.04$, $95 \% C I=-0.12$ to $0.20, P=0.61$, for DES-ISR; and $M D=-0.06$, 95\% CI $=-0.35$ to $0.24, P=0.71$ for BMS-ISR, Table 3). Sensitive analysis by leave-one-out approach showed the overall result remained stable of our study. The TSA indicated more studies were needed to verify the result (Data not shown).

3.2.3. Percent Diameter Stenosis. All six trials compared the percent diameter stenosis between patients treated with DCB and those treated with EES (569 versus 565 patients, respectively). From the synthetic result, the DCB was associated with higher percent diameter stenosis with a level of MD 5.37 $\left(M D=5.37,95 \% C I=1.33\right.$ to $9.42, P=0.009, I^{2}=68 \%$, Figure 2$)$. There were significant heterogeneities among trials with $I^{2}=68 \%$. For the subgroup meta-analysis, compared with EES, DCB was associated with higher percent diameter stenosis only 


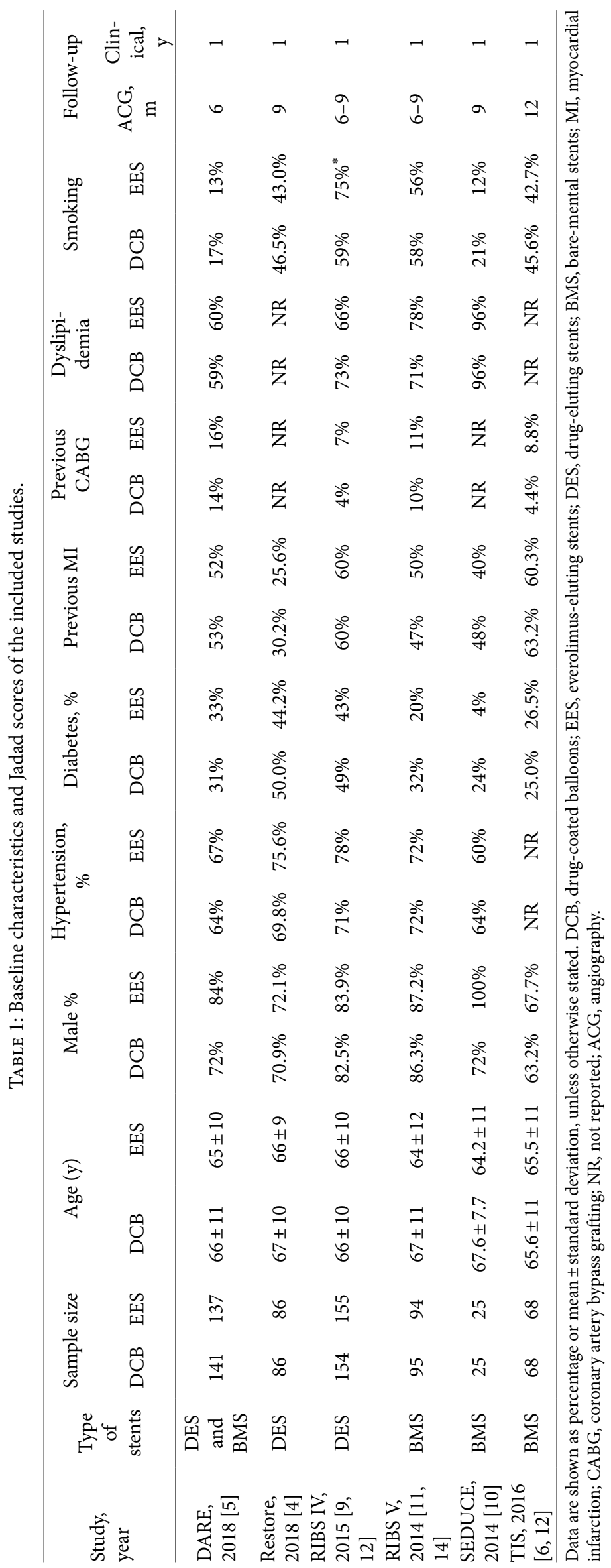




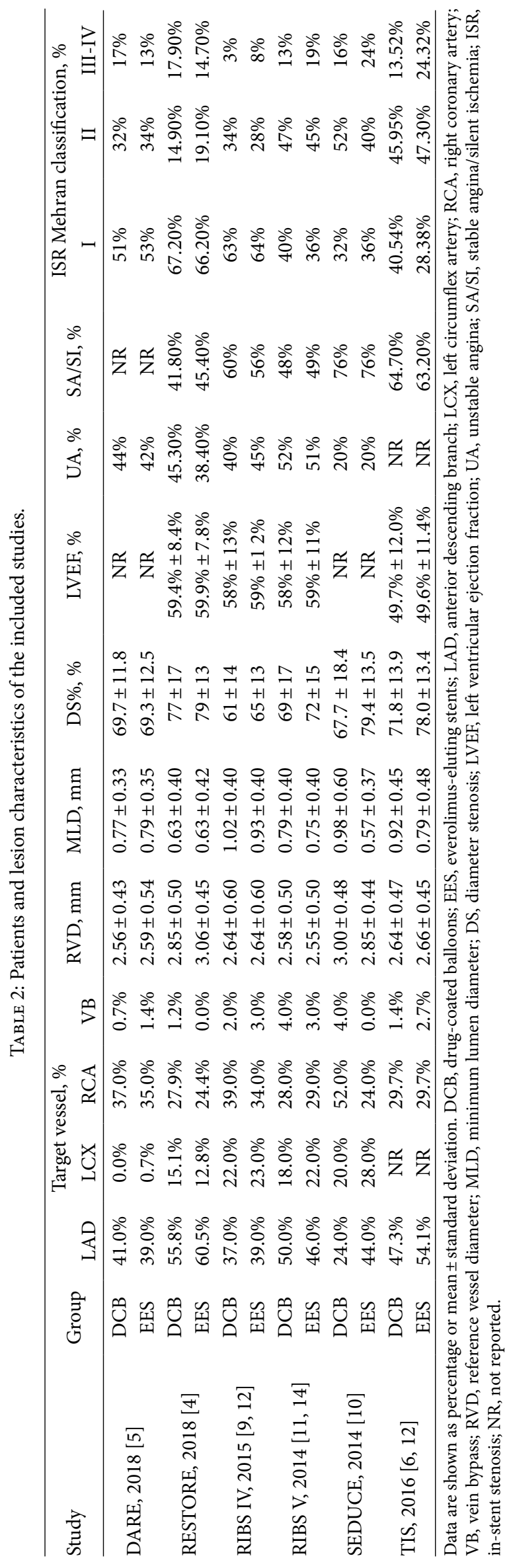




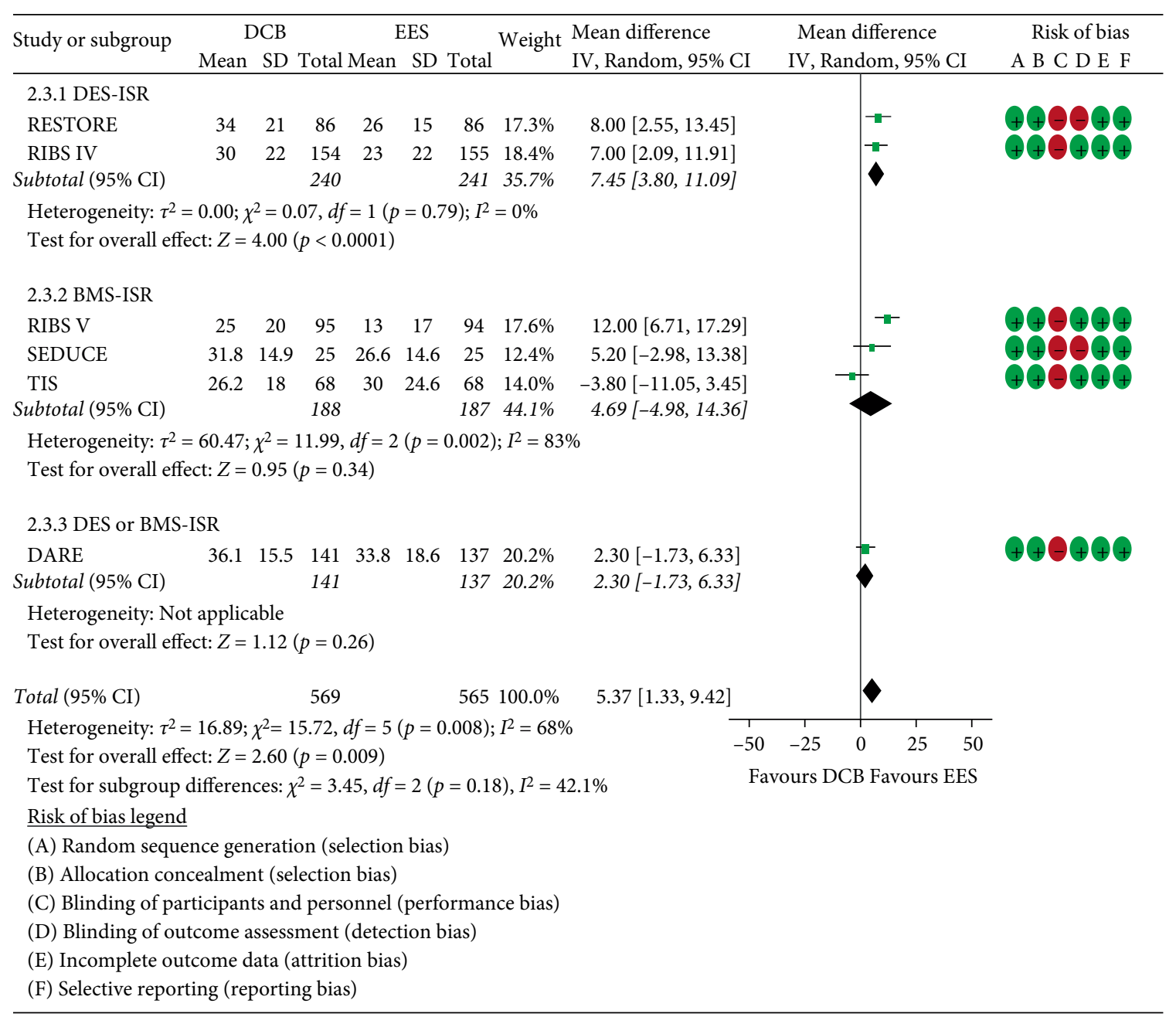

FIGURE 2: Mean difference in the percent diameter stenosis between the DCB and EES groups, and the risk of bias among included studies. Subgroup analysis was performed based on the type of restenosed stent. BMS, bare-metal stents; DES, drug-eluting stents; DCB, drug-coated balloons; EES, everolimus-eluting stents.

TABLE 3: Summary of the main results.

\begin{tabular}{|c|c|c|c|c|c|c|}
\hline \multirow[b]{2}{*}{ Items } & \multicolumn{6}{|c|}{ Population } \\
\hline & $N 1$ & $\begin{array}{l}\text { DES-ISR: pooled effect } \\
\text { size DCB:EES }(95 \% \mathrm{CI})\end{array}$ & $N 2$ & $\begin{array}{l}\text { BMS-ISR: pooled effect } \\
\text { size DCB:EES }(95 \% \mathrm{CI})\end{array}$ & $N(N 1+N 2)$ & $\begin{array}{l}\text { Overall: pooled effect size } \\
\text { DCB:EES (95\%CI) }\end{array}$ \\
\hline MLD & 481 & $M D=-0.25(-0.37,-0.14)^{*}$ & 375 & $M D=-0.15(-0.39,0.09)$ & 1134 & $M D=-0.17(-0.29,-0.05)^{*}$ \\
\hline LLL & 481 & $M D=0.04(-0.12,0.20)$ & 375 & $M D=-0.06(-0.35,0.24)$ & 1134 & $M D=-0.06(-0.23,0.10)$ \\
\hline Percent diameter stenosis & 481 & $M D=7.45(3.80,11.09)^{*}$ & 375 & $M D=4.69(-4.98,14.36)$ & 1134 & $M D=5.37(1.33,9.42)^{*}$ \\
\hline Binary restenosis & 481 & $R R=2.07(1.20,3.58)^{*}$ & 375 & $R R=0.89(0.47,1.68)$ & 1134 & $R R=0.77(0.44,1.34)$ \\
\hline TLR & 481 & $R R=2.43(1.28,4.62)^{*}$ & 239 & $R R=2.23(0.70,7.10)$ & 720 & $R R=2.38(1.36,4.18)^{*}$ \\
\hline TVR & 481 & $R R=2.07(1.22,3.50)^{*}$ & 375 & $R R=0.82(0.46,1.46)$ & 1134 & $R R=1.17(0.52,2.61)$ \\
\hline Myocardial infarction & 481 & $R R=1.01(0.22,4.73)$ & 375 & $R R=0.80(0.31,2.06)$ & 1134 & $R R=0.91(0.48,1.73)$ \\
\hline Death & 481 & $R R=1.10(0.50,2.41)$ & 375 & $R R=1.45(0.62,3.36)$ & 1134 & $R R=1.19(0.68,2.07)$ \\
\hline
\end{tabular}

DCB, drug-coated balloons; EES, everolimus-eluting stents; DES, drug-eluting stents; BMS, bare-mental stents; MLD, minimum lumen diameter; LLL, late lumen loss; TLR, target lesion revascularization; TVR, target vessel revascularization; $\mathrm{MD}$, mean difference; $\mathrm{RR}$, risk ratio; $\mathrm{CI}$, confidence interval. ${ }^{*} P<0.05$.

for DES-ISR $(M D=7.45,95 \% C I=3.80$ to $11.09, P<0.001$, Figure 2), but not for BMS-ISR $(M D=4.69,95 \% C I$ $=-4.98$ to $14.36, P=0.34$, Figure 2 ). Exclusion of any single trial from the analysis (leave-one-out meta-analysis) did not substantively alter the overall result of our analysis. The TSA indicated the meta-analysis became conclusive according to the O'Brien-Fleming boundaries after the cumulative significance testing (Figure 3(a)). 


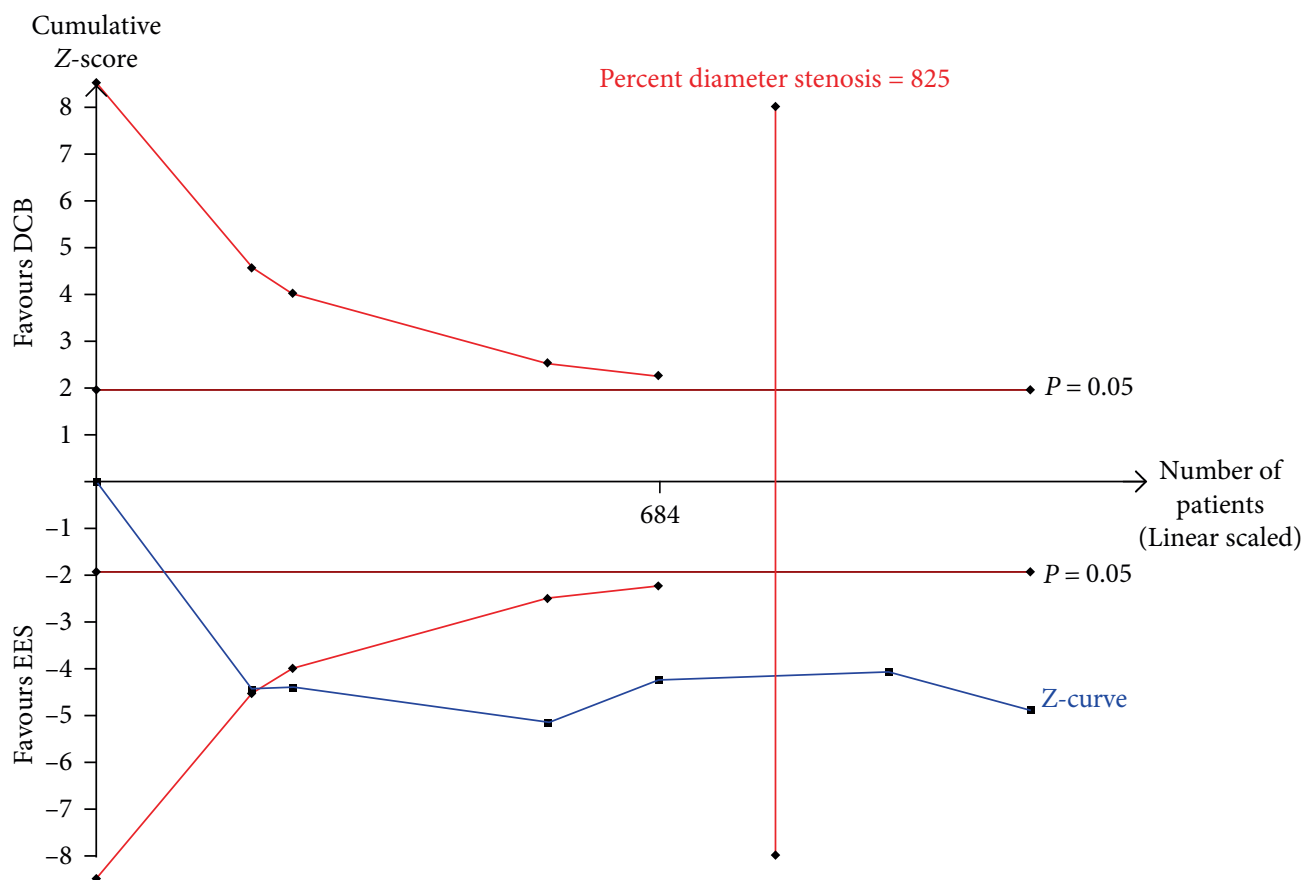

(a)

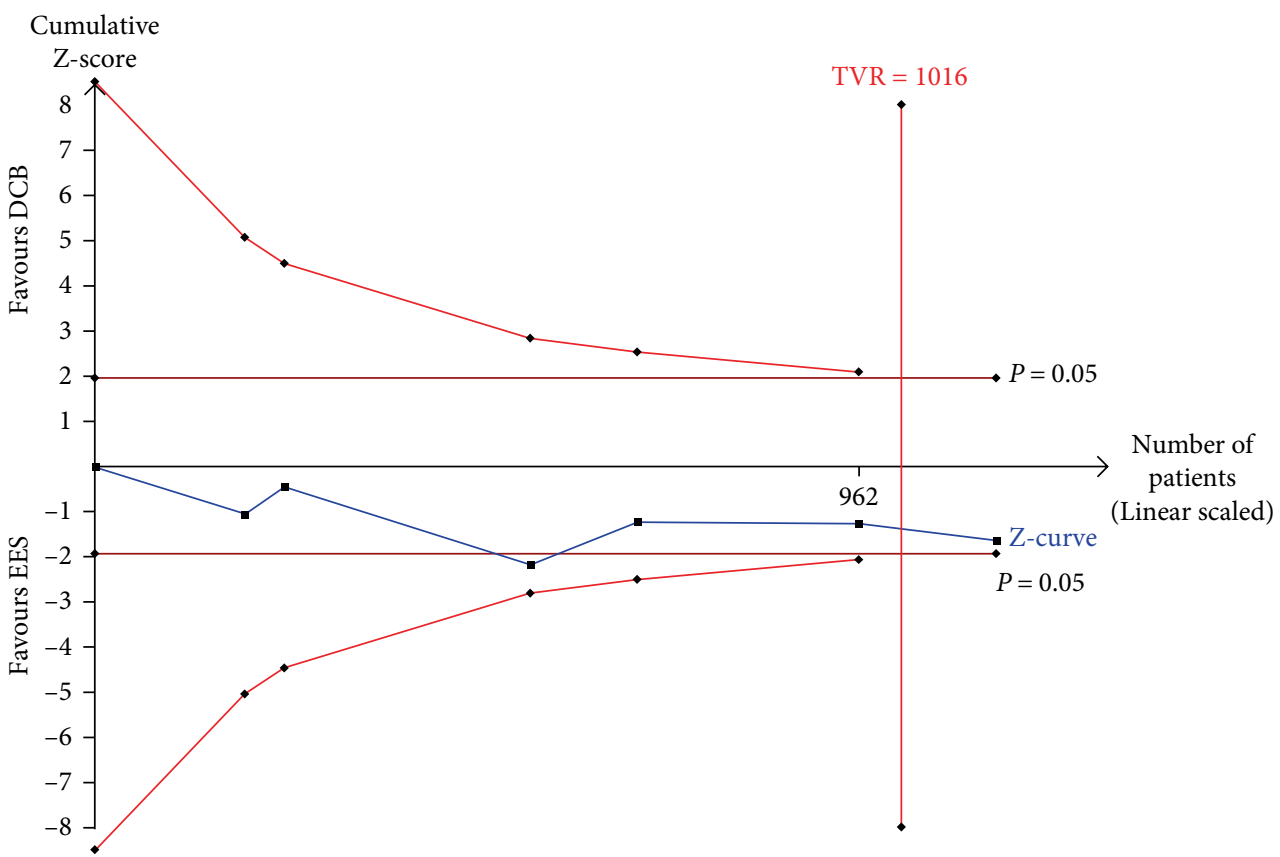

(b)

FIGURE 3: The trial sequential meta-analysis (TSA) of DCB versus EES for the treatment of in-stent restenosis on percent diameter stenosis (a) and target vessel revascularization (b). DCB, drug-coated balloons; EES, everolimus-eluting stents.

3.2.4. Binary Restenosis. All six trials reported binary restenosis between DCB and EES groups. A total of 70 binary restenosis were identified in the DCB group with 569 patients, while 59 binary restenosis were identified in the EES groups with 565 patients. The pooled result showed that binary restenosis did not differ between the DCB and EES groups (pooled $R R=1.25,95 \% C I=0.68$ to $2.27, P=0.47, I^{2}=58 \%$, Table 3 and Figure 4). The subgroup analyses revealed that the incidence of binary restenosis was 2.01-fold higher in the DCB group for DES-ISR patients (pooled $R R=2.07,95 \% C I=1.20$ to $3.58, P=0.009$, Table 3 and Figure 4). While for BMS-ISR patients, the incidence of binary restenosis was similar between the groups (pooled $R R=1.03,95 \% C I=0.34$ to $3.14, P=0.96$, Table 3 and Figure 4). The pooled results were robust to the deletion of individual studies, either among the whole group or within each subgroup. The meta-analysis becomes conclusive according to the TSA. 


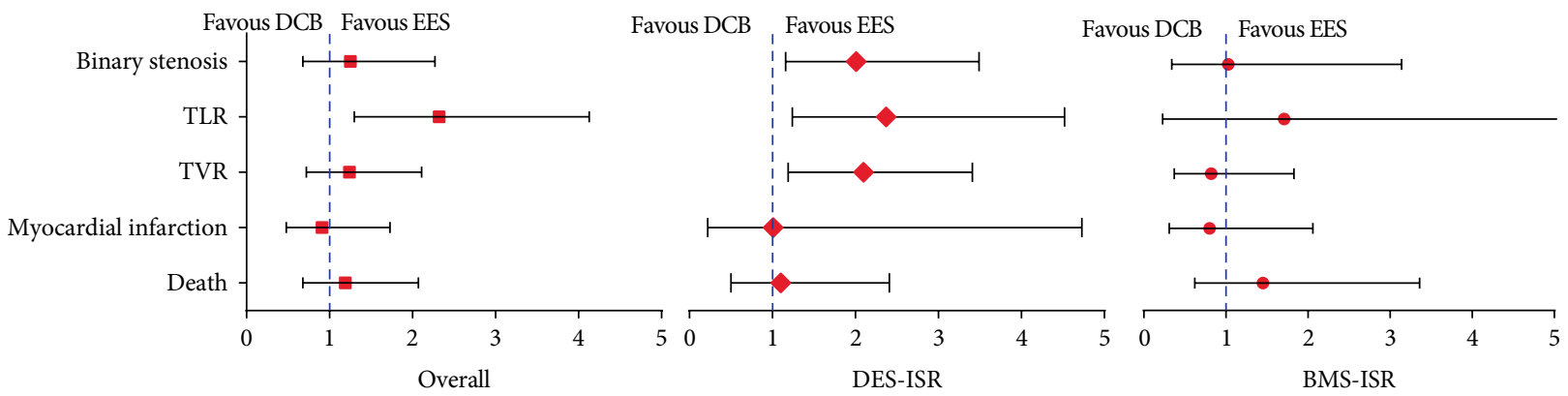

FIGURE 4: Summary of main event comparisons between DCB and EES across subgroups. BMS, bare-metal stents; DES, drug-eluting stents; DCB, drug-coated balloons; EES, everolimus-eluting stents.

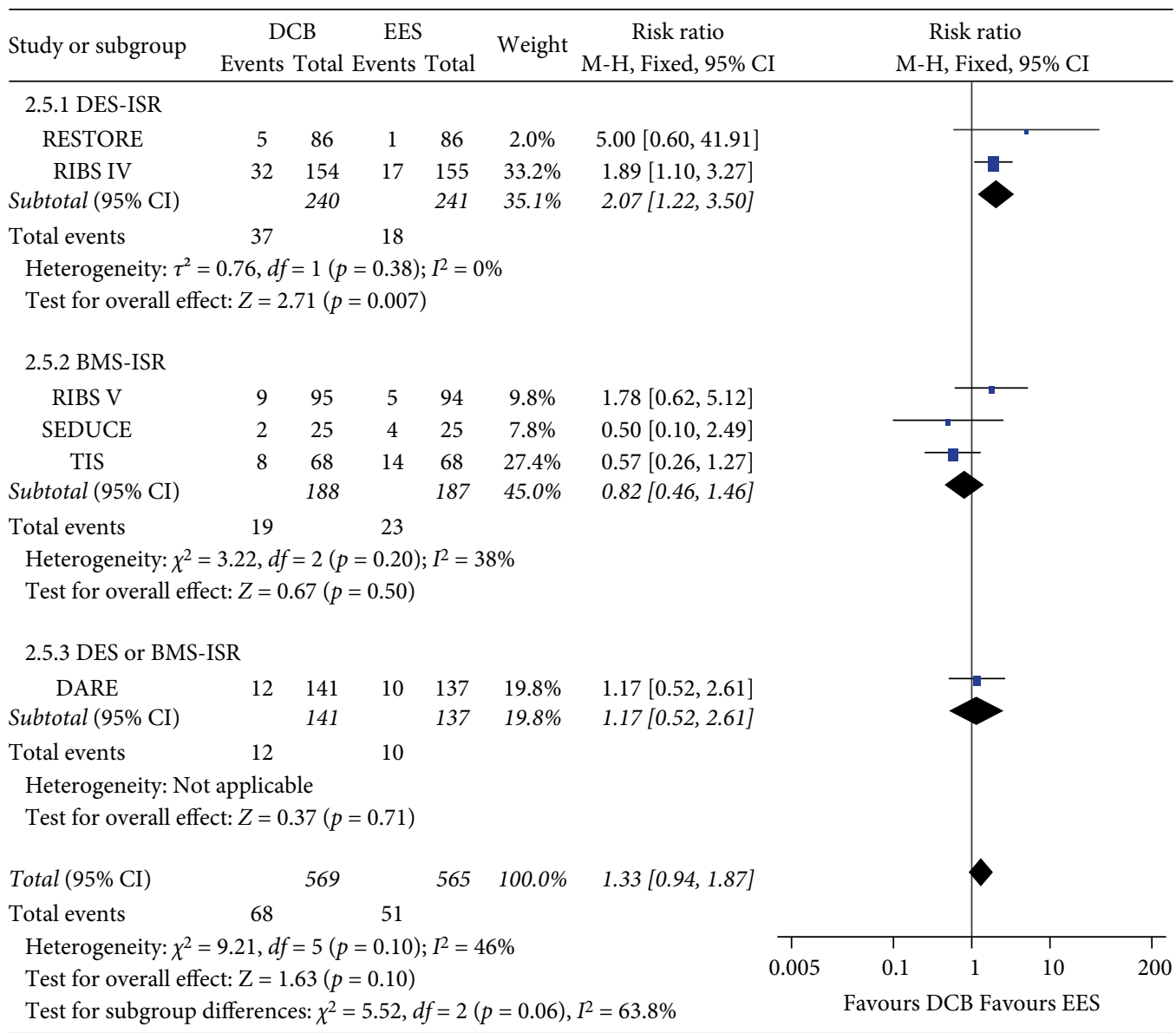

FIGURE 5: Comparison of the risk of TVR between DCB and EES. Subgroup analysis was performed based on the type of restenosed stent. BMS, bare-metal stents; DES, drug-eluting stents; DCB, drug-coated balloons; EES, everolimus-eluting stents; TVR, target vessel revascularization.

3.3. Clinical Events. With respect to clinical events, three trials reported 1-year clinical endpoints and another three trials reported 3-year clinical outcomes. All six included studies reported TVR in detail. The overall meta-analysis showed that the incidence of TVR did not differ between the groups (Fixed-effect model $R R=1.33,95 \% C I=0.94$ to $1.87, P=0.44, I^{2}=46 \%$, Figure 5). However, for DES-ISR, there was a 2.07-fold higher TVR in the DCB group than in the EES group $(R R=2.07,95 \% C I=1.22$ to $3.50, P=0.007$, Figure 5$)$.
For BMS-ISR, the incidence of TVR was comparable between the DCB and EES groups $(R R=0.82,95 \% C I=$ 0.46 to $1.46, P=0.50$, Figure 5$)$. However, the overall result became significant on deletion of the TIS trial $(R R=1.61$, $95 \% C I=1.10$ to $2.38, P=0.02)$. The TSA indicated the result is conclusive based on the O'Brien-Fleming boundaries (Figure 3(b)).

Only four trials were eligible for the pooled analysis of TLR. Of 360 patients, 38 (10.6\%) patients in the DCB group experienced TLR, while 16 (4.4\%) of 360 patients in the EES 
group received TLR. Pooled analysis using a fixed-effects model showed that DCB had significantly higher TLR than EES $\left(R R=2.38,95 \% C I=1.36\right.$ to $4.18, P=0.002, I^{2}=0 \%$, Table 3 and Figure 4). Subgroup analyses revealed that DCB increased TLR only in patients with DES-ISR $(R R=2.43$, $95 \% C I=1.28$ to $4.22, P=0.002$, Table 3 and Figure 4$)$ and not in patients with BMS-ISR $(R R=2.23,95 \% C I=0.70$ to 7.10 , $P=0.17$, Table 3 and Figure 4$)$. Sensitivity analysis were not conducted since there are only 4 trials.

No statistically significant differences in myocardial infarction and death in both patients with DES-ISR and BMSISR (Table 3 and Figure 4), but more studies were needed to verify the results according to TSA. The direction of the results remained unchanged when removing any single trial.

\section{Discussion}

The present pair-wise meta-analysis included six RCTs with 1134 patients and compared DCB to EES for ISR treatment. The pooled results showed that DCB had differential relative efficacy between DES-ISR and BMS-ISR compared with EES. For DES-ISR, DCB was inferior to EES both on angiographic results and clinical events. In detail, DCB had lower MLD, higher percent diameter stenosis, more binary restenosis, and higher incidence of TVR and TLR than EES in DES-ISR patients. However, in BMS-ISR patients, the efficacy of EES and DCB in terms of angiographic results and clinical events were comparable.

DCB was first introduced in 2006 for the clinical treatment of ISR as it does not require implanting additional metal layers for drug release [15]. Thereafter, many randomized trials attempted to evaluate the efficacy of DCB for the treatment of ISR. Nowadays, robust evidences showed that DCB was superior to uncoated balloon angioplasty for the treatment of DESISR and BMS-ISR [16]. With increasing evidences, DCB is an established treatment option of DES-ISR and BMS-ISR with a Class I, Level of Evidence: a recommendation in the European guidelines, the same as DES [2]. However, the efficacy between DCB and DES for the treatment of ISR is still unknown.

Randomized trials have demonstrated that DCB is associated with comparable angiographic results and clinical events with first-generation DES $[17,18]$. EES was a second-generation DES, which was superior to BMS and first-generation DES in reducing the risk of stent thrombosis and repeat revascularization $[1,19]$. Several trials compared DCB with EES for the treatment of ISR, but they had small sample sizes and inconsistent outcomes [4, 5, 8-11]. Thus, the clinical outcomes on the comparison of DCB and EES for the treatment of ISR were underpowered.

The present meta-analysis showed differential relative efficacy between DCB and EES in DES-ISR and BMS-ISR patients. DCB had comparable angiographic results and clinical events with EES in BMS-ISR patients. However, for DES-ISR patients, DCB was inferior to EES in terms of both angiographic results and clinical events. This situation has significant clinical implications. Currently, in the United States, more than $80 \%$ of stents implanted during PCI were DES [20]. In China, the proportion of DES use was up to $99.6 \%$ for stent implantation
[21]. Even in patients with second-generation DES implantation, the incidence of ISR is higher than $10 \%$, and the rate of repeat revascularization for DES-ISR is still encountered in $5-10 \%$ of patients undergoing percutaneous coronary intervention [22]. In other words, DES-ISR is the majority type of ISR in clinical practice, especially in China. Therefore, it is very important to clarify the treatment strategies of DES-ISR and BMS-ISR. In the latest European myocardial revascularization guidelines, both DES and DCB were recommended for the treatment of DES-ISR or BMS-ISR (Class I, Level A) [2]. The inferiority of DCB to EES for the treatment of DESISR in the present meta-analysis arisen a challenge for DCB treatment in DES-ISR patients and indicated expectation of more high-quality trials to further evaluate the efficacy of DCB for the treatment of DES-ISR.

Previous network meta-analysis showed that EES was more effective for the treatment of ISR compared with DCB, with the lowest risks of restenosis and repeat revascularization compared with other treatments. However, this network meta-analysis only include two head-to-head comparative trials, RIBS IV and RIBS V, to synthesize the direct result of EES versus DCB. Differed from the network meta-analysis, our study included six head-to-head trials, and the results from subgroup analyses indicated that EES was inferior to DCB only in DES-ISR, but not in BMS-ISR.

Previous studies had demonstrated that DCB angioplasty was more effective in BMS-ISR than in DES-ISR, with no difference on the type of DES [16, 23]. A prospective, multicenter, randomized trial conducted by Habara et al. on 208 patients showed that DCB reduced neointimal hyperplasia more effectively in BMS-ISR than in DES-ISR at 6 months angiographic and clinical follow-up after intervention [16]. SeQuent Please World Wide Registry showed that the TLR rate was significantly lower in patients with $\mathrm{DCB}$ angioplasty for BMS-ISR than in those for DES-ISR [23]. In the present study, the finding of differential relative efficacy for DCB between BMS-ISR and DES-ISR is consistent with the finding of these studies.

Several potential mechanisms are responsible for the varying efficacies of DCB on DES-ISR and BMS-ISR. First, the histomorphological features of neointimal differed in BMSISR and DES-ISR patients. Nakano et al. carried out a human autopsy registry and indicated that neointimal compositions of DES-ISR demonstrated greater proteoglycan deposition and less smooth muscle cellularity over time, compared with BMSISR with greater smooth muscle cell density and collagen deposition [24]. In the present meta-analysis, paclitaxel-coated balloons were used in all included trials. A previous study demonstrated that paclitaxel reduced in-stent intimal hyperplasia by inhibiting arterial smooth muscle cell proliferation and migration [25]. Therefore, the BMS-ISR with greater smooth muscle cell density might have higher drug efficacy. Second, DES-ISR might already have the drug resistance or local hypersensitivity reactions, whereas BMSISR is still naive regarding the treatment [26]. Third, ISR occurs earlier in patients implanted with BMS, and neointimal hyperplasia rich in smooth muscle cells is the prevalent mechanism [27]. While in patients with DES implantation, as the effects of antiproliferative drugs eluted by the stent 
impaired physiological reendothelialization and vascular remodeling, chronic inflammation, and delayed and aberrant arterial healing were frequently observed in-stent of DES, which induced the formation of neoatherosclerosis [27]. Delayed and abnormal arterial healing, persistent inflammatory process, and/or incompetent endothelial function might decrease the efficacy of DCB in DES-ISR patients [28].

This study had some potential limitations. First, paclitaxelcoated balloons were exclusively used DCB in all the included trials. Therefore, our findings only reflected the effect of paclitaxel-coated balloons for the treatment of ISR. Recently, a novel sirolimus-coated balloon was investigated in DES-ISR patients compared with paclitaxel-coated balloons [29]. The efficacy of the novel DCB for the treatment DES-ISR is still an ongoing exploration. Second, our findings need to be considered as average effects of DES-ISR, because detailed types of DES-ISR were not available in the present study. Given the similar mechanisms underlying different DES-ISR [27], the generalizability of our results seems viable. Third, while this type of analysis has particular shortcomings when discussing efficacy between EES and DEB such as different drug coatings, different stent platforms, and so on, the results of this analysis can be generalizable due to shared pathophysiology that contributes to in-stent restenosis. Fourth, consistent heterogeneity was observed for the angiographic results among included studies. Random-effects model was used to account for the heterogeneity. Fifth, the inherent limitations of meta-analyses cannot be ignored, such as publication bias.

\section{Conclusions}

DCB has differential relative efficacy between BMS-ISR and DES-ISR. In DES-ISR patients, DCB was associated with lower MLD, higher percent diameter stenosis, more binary stenosis, and higher rate of TVR and TLR. However, for BMS-ISR, no significant difference was found between DCB and EES. More high-quality randomized trials are needed to further evaluate the role of DCB for the treatment of ISR, especially in patients with DES-ISR. The potential mechanisms of the varying efficacy between BMS-ISR and DES-ISR for DCB need to be further investigated.

\section{Abbreviations}

DCB: drug-coated balloons

EES: everolimus-eluting stents

ISR: in-stent restenosis

DES: drug-eluting stents

BMS: bare-mental stents

MLD: minimum lumen diameter

TVR: target vessel revascularization

TLR: target lesion revascularization

LLL: late lumen loss.

\section{Conflicts of Interest}

The authors declare that they have no conflicts of interest.

\section{Authors' Contributions}

The first two authors contributed equally to this manuscript.

\section{Funding}

This work was supported by Guizhou Provincial Science and Technology Foundation (No. [2019]1197) and National Clinical Key Specialty Construction Project of China [No. (2013) 544]. There are no relationships with industry.

\section{Acknowledgments}

We would like to thank Editage (https://app.hindawi.editage. com) for English language editing.

\section{References}

[1] R. Piccolo, K. H. Bonaa, O. Efthimiou et al., "Drug-eluting or bare-metal stents for percutaneous coronary intervention: a systematic review and individual patient data meta-analysis of randomised clinical trials," The Lancet, vol. 393, no. 10190, pp. 2503-2510, 2019.

[2] F. J. Neumann, M. Sousa-Uva, A. Ahlsson et al., "2018 ESC/ EACTS guidelines on myocardial revascularization," European Heart Journal, vol. 40, no. 2, pp. 87-165, 2019.

[3] G. C. Siontis, G. G. Stefanini, D. Mavridis et al., "Percutaneous coronary interventional strategies for treatment of in-stent restenosis: a network meta-analysis," The Lancet, vol. 386, no. 9994, pp. 655-664, 2015.

[4] Y. Wong, D. Y. Kang, J. B. Lee et al., "Comparison of drug-eluting stents and drug-coated balloon for the treatment of drug-eluting coronary stent restenosis: a randomized RESTORE trial," American Heart Journal, vol. 197, pp. 35-42, 2018.

[5] J. J. Baan, B. E. Claessen, K. B. Dijk et al., "A randomized comparison of paclitaxel-eluting balloon versus everolimuseluting stent for the treatment of any in-stent restenosis: the DARE trial," JACC Cardiovascular Interventions, vol. 11, no. 3, pp. 275-283, 2018.

[6] L. Pleva, P. Kukla, P. Kusnierova, J. Zapletalova, and O. Hlinomaz, "Comparison of the efficacy of paclitaxel-eluting balloon catheters and everolimus-eluting stents in the treatment of coronary in-stent restenosis: the treatment of in-stent restenosis study," Circulation Cardiovascular Interventions, vol. 9, no. 4, p. e3316, 2016.

[7] D. Moher, A. Liberati, J. Tetzlaff, and D. G. Altman, "Preferred reporting items for systematic reviews and meta-analyses: the PRISMA statement," Journal of Clinical Epidemiology, vol. 62, no. 10, pp. 1006-1012, 2009.

[8] W. Liu, X. Yang, and J. Huang, "Efficacy and safety of insulin degludec versus insulin glargine: a systematic review and 
meta-analysis of fifteen clinical trials," International Journal of Endocrinology, vol. 2018, pp. 1-10, 2018.

[9] F. Alfonso, M. J. Perez-Vizcayno, A. Cardenas et al., "A prospective randomized trial of drug-eluting balloons versus everolimus-eluting stents in patients with in-stent restenosis of drug-eluting stents: the RIBS IV randomized clinical trial," Journal of the American College of Cardiology, vol. 66, no. 1, pp. 23-33, 2015.

[10] T. Adriaenssens, J. Dens, G. Ughi et al., "Optical coherence tomography study of healing characteristics of paclitaxel-eluting balloons vs. everolimus-eluting stents for in-stent restenosis: the SEDUCE (Safety and efficacy of a Drug elUting balloon in Coronary artery rEstenosis) randomised clinical trial," EuroIntervention, vol. 10, no. 4, pp. 439-448, 2014.

[11] F. Alfonso, M. J. Perez-Vizcayno, A. Cardenas et al., "A randomized comparison of drug-eluting balloon versus everolimus-eluting stent in patients with bare-metal stent-instent restenosis: the RIBS V Clinical Trial (Restenosis Intra-stent of Bare Metal Stents: paclitaxel-eluting balloon vs. everolimuseluting stent)," Journal of the American College of Cardiology, vol. 63, no. 14, pp. 1378-1386, 2014.

[12] F. Alfonso, M. J. Perez-Vizcayno, J. Cuesta et al., "3-year clinical follow-up of the RIBS IV clinical trial: a prospective randomized study of drug-eluting balloons versus everolimuseluting stents in patients with in-stent restenosis in coronary arteries previously treated with drug-eluting stents," JACC Cardiovascular Interventions, vol. 11, no. 10, pp. 981-991, 2018.

[13] L. Pleva, P. Kukla, J. Zapletalova, and O. Hlinomaz, "Long-term outcomes after treatment of bare-metal stent restenosis with paclitaxel-coated balloon catheters or everolimus-eluting stents: 3-year follow-up of the TIS clinical study," Catheterization and Cardiovascular Interventions, vol. 92, no. 6, pp. E416-E424, 2018.

[14] F. Alfonso, M. J. Perez-Vizcayno, D. B. B. Garcia et al., "Longterm results of everolimus-eluting stents versus drug-eluting balloons in patients with bare-metal in-stent restenosis: 3-year follow-up of the RIBS V clinical trial," JACC Cardiovascular Interventions, vol. 9, no. 12, pp. 1246-1255, 2016.

[15] B. Scheller, C. Hehrlein, W. Bocksch et al., "Treatment of coronary in-stent restenosis with a paclitaxel-coated balloon catheter," The New England Journal of Medicine, vol. 355, no. 20, pp. 2113-2124, 2006.

[16] S. Habara, M. Iwabuchi, N. Inoue et al., "A multicenter randomized comparison of paclitaxel-coated balloon catheter with conventional balloon angioplasty in patients with baremetal stent restenosis and drug-eluting stent restenosis," American Heart Journal, vol. 166, no. 3, pp. 527-533, 2013.

[17] B. Xu, R. Gao, J. Wang et al., "A prospective, multicenter, randomized trial of paclitaxel-coated balloon versus paclitaxeleluting stent for the treatment of drug-eluting stent in-stent restenosis: results from the PEPCAD China ISR trial," JACC Cardiovascular Interventions, vol. 7, no. 2, pp. 204-211, 2014.

[18] R. A. Byrne, F. J. Neumann, J. Mehilli et al., "Paclitaxel-eluting balloons, paclitaxel-eluting stents, and balloon angioplasty in patients with restenosis after implantation of a drug-eluting stent (ISAR-DESIRE 3): a randomised, open-label trial," The Lancet, vol. 381, no. 9865, pp. 461-467, 2013.

[19] M. Valgimigli, M. Tebaldi, M. Borghesi et al., "Two-year outcomes after first- or second-generation drug-eluting or bare-metal stent implantation in all-comer patients undergoing percutaneous coronary intervention: a pre-specified analysis from the PRODIGY study (PROlonging Dual Antiplatelet Treatment
After Grading stent-induced Intimal hyperplasia studY)," JACC Cardiovascular Interventions, vol. 7, no. 1, pp. 20-28, 2014.

[20] E. J. Benjamin, P. Muntner, A. Alonso et al., "Heart disease and stroke statistics-2019 update: a report from the American Heart Association," Circulation, vol. 139, no. 10, pp. e56-e528, 2019.

[21] S. Hu, Report of cardiovascular disease in China 2017, China Encyclopedia Publishing House, Beijingp. 126, 2018.

[22] S. Cassese, R. A. Byrne, T. Tada et al., "Incidence and predictors of restenosis after coronary stenting in 10004 patients with surveillance angiography," Heart, vol. 100, no. 2, pp. 153-159, 2014.

[23] J. Wohrle, M. Zadura, S. Mobius-Winkler et al., "SeQuent Please World Wide Registry: clinical results of SeQuent please paclitaxel-coated balloon angioplasty in a large-scale, prospective registry study," Journal of the American College of Cardiology, vol. 60, no. 18, pp. 1733-1738, 2012.

[24] M. Nakano, F. Otsuka, K. Yahagi et al., "Human autopsy study of drug-eluting stents restenosis: histomorphological predictors and neointimal characteristics," European Heart Journal, vol. 34, no. 42, pp. 3304-3313, 2013.

[25] D. I. Axel, W. Kunert, C. Goggelmann et al., "Paclitaxel inhibits arterial smooth muscle cell proliferation and migration in vitro and in vivo using local drug delivery," Circulation, vol. 96, no. 2, pp. 636-645, 1997.

[26] F. Alfonso, R. A. Byrne, F. Rivero, and A. Kastrati, "Current treatment of in-stent restenosis," Journal of the American College of Cardiology, vol. 63, no. 24, pp. 2659-2673, 2014.

[27] J. A. Borovac, D. D'Amario, R. Vergallo et al., "Neoatherosclerosis after drug-eluting stent implantation: a novel clinical and therapeutic challenge," European Heart Journal Cardiovascular Pharmacotherapy, vol. 5, no. 2, pp. 105-116, 2019.

[28] S. J. Park, S. J. Kang, R. Virmani, M. Nakano, and Y. Ueda, "Instent neoatherosclerosis: a final common pathway of late stent failure," Journal of the American College of Cardiology, vol. 59, no. 23, pp. 2051-2057, 2012.

[29] R. M. Ali, K. M. Abdul, A. W. Wan et al., "Treatment of coronary drug-eluting stent restenosis by a sirolimus- or paclitaxel-coated balloon," JACC Cardiovascular Interventions, vol. 12, no. 6, pp. 558-566, 2019. 


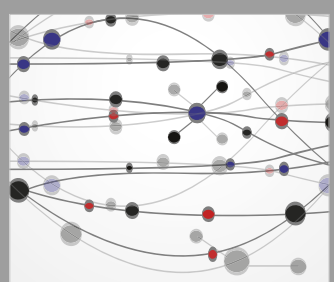

The Scientific World Journal
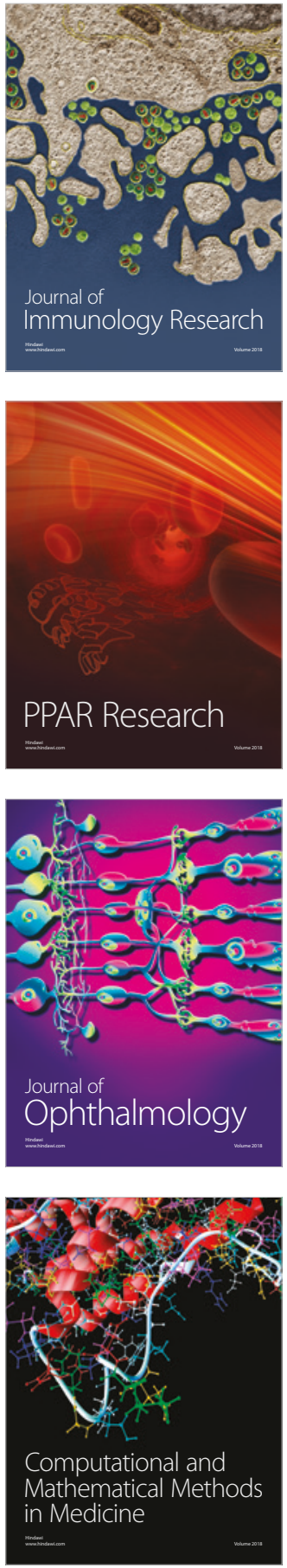

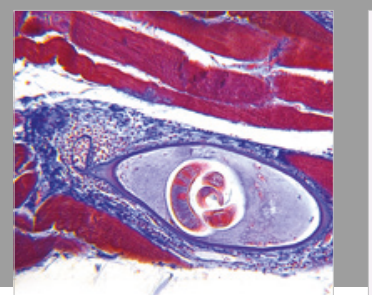

Gastroenterology Research and Practice

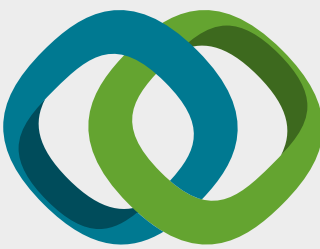

\section{Hindawi}

Submit your manuscripts at

www.hindawi.com
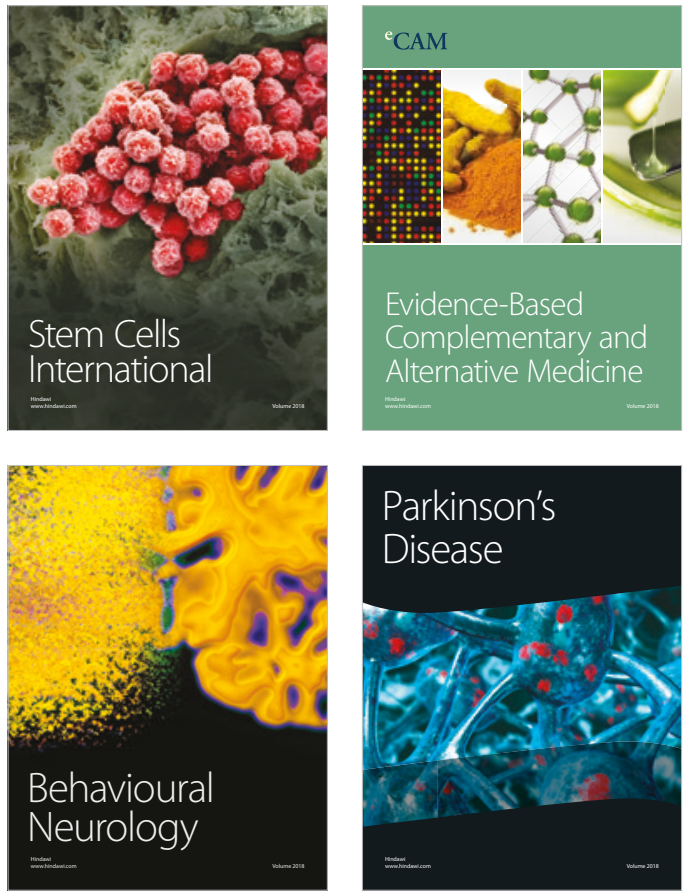

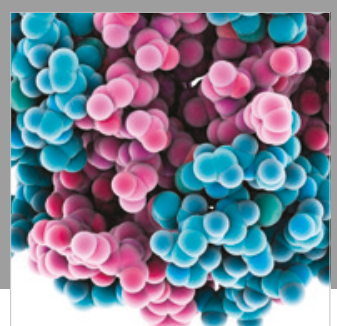

ournal of

Diabetes Research

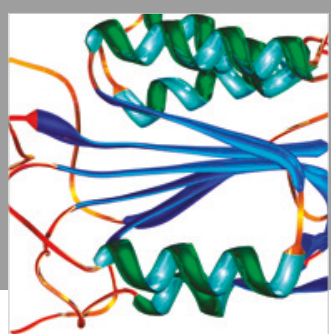

Disease Markers
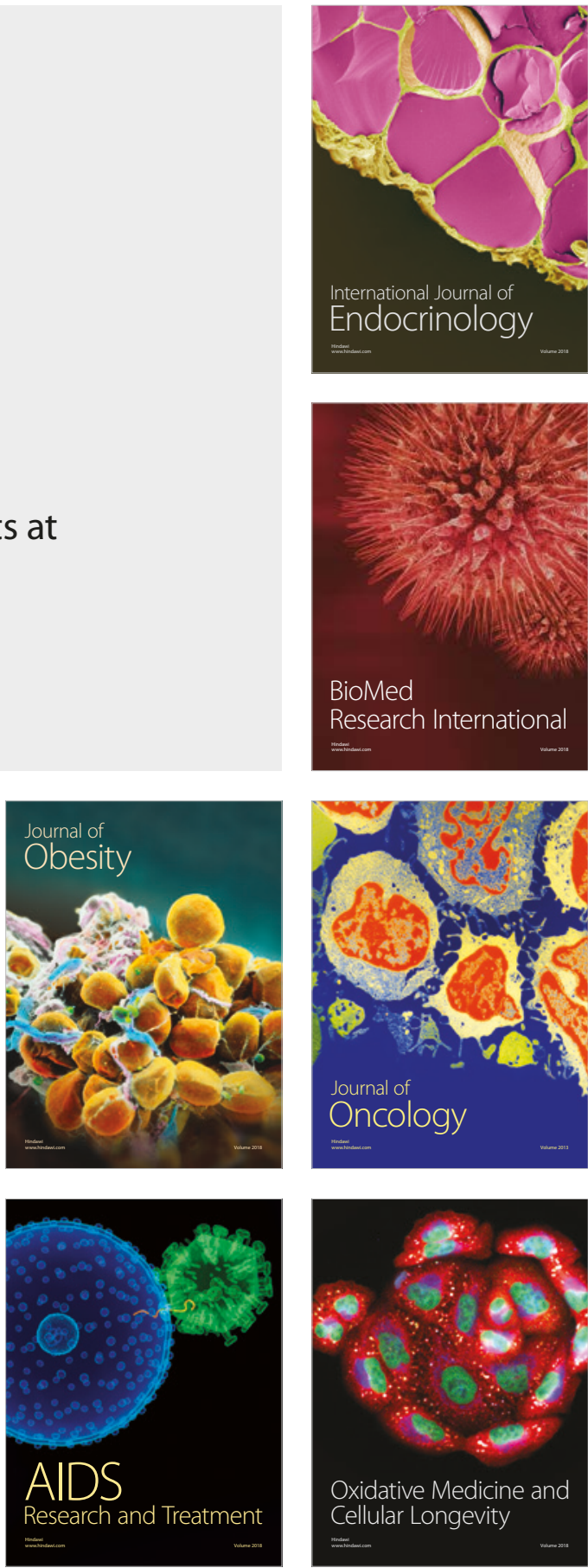